\title{
Approximation of Function and Its Derivative by the Modificated Steklov Operator
}

\begin{abstract}
A. A. Khromov
Saratov State University, 83, Astrakhanskaya str., Saratov, 410012, Russia, KhromovAP@info.sgu.ru

With the use of modification of Steklov operator are constructed families of integral operator which allow us to get uniform derivative on a closed.
\end{abstract}

Key words: derivative, uniform approximations, Steklov operator.

This work was supported by the Russian Foundation for Basic Research (project no. 13-01-00238).

\section{References}

1. Ivanov V. K. Ob integral'nykh uravneniiakh Fredgol'ma I roda [Fredholm integral equation of the first kind]. Differents. uravneniia [Differ. Equations], 1967, vol. III, no. 3, pp. 410-421 (in Russian).

2. Khromov A. P., Khromova G. V. Ob odnoi modifikatsii operatora Steklova [One modification of the Steklov operator]. Souremennye problemy teorii funktsii $i$ ikh prilozheniia: Tez. dokl. 15-i Sarat. zimn. shkoly [Modern problems of function theory and their applications: abstracts of the 15-th Saratov winter school], Saratov, Saratov Univ. Press, 2010, pp. 181 (in Russian).

3. Khromova G. V. Error estimates of approximate solutions to equations of the first kind. Doklady Math.. 2001, vol. 63 , no. 3, 390-394.

УДК 517.51:571.968

\section{РЕГУЛЯРИЗАЦИЯ УРАВНЕНИЯ АБЕЛЯ С ПОМОЩЬЮ РАЗРЫВНОГО ОПЕРАТОРА СТЕКЛОВА}

\section{Г. В. Хромова}

Доктор фризико-математических наук, просессор кафредры математической фризики и вычислительной математики, Саратовский государственный университет им. Н. Г. Чернышевского, KhromovAP@info.sgu.ru

Для нахождения равномерных приближений к точному решению уравнения Абеля с приближенно заданной правой частью предложено простое по конструкции семейство интегральных операторов.

Ключевые слова: уравнение Абеля, оператор Стеклова, равномерные приближения, отрезок.

1. Рассмотрим уравнение Абеля:

$$
A u \equiv \int_{0}^{x} \frac{(x-t)^{\beta-1}}{\Gamma(\beta)} u(t) d t=f(x), \quad 0<\beta<1, \quad 0 \leq x \leq 1 .
$$

Пусть известно, что при данной $f(x)$ существует непрерывная функция $u(x)$, являющаяся решением уравнения (1), но сама функция $f(x)$ нам неизвестна - вместо нее известна $f_{\delta}(x)$ такая, что $\left\|f_{\delta}-f\right\|_{L_{2}} \leq \delta$. Поставим задачу: по $f_{\delta}(x)$ и $\delta$ найти равномерные приближения к $u(x)$.

Возьмем разрывный оператор Стеклова из [1]:

$$
S_{\alpha} u= \begin{cases}\frac{1}{\alpha} \int_{x}^{x+\alpha} u(t) \mathrm{dt}, \quad x \in[0,1 / 2] \\ \frac{1}{\alpha} \int_{x-\alpha}^{x} u(t) \mathrm{dt}, \quad x \in[1 / 2,1] .\end{cases}
$$

По методу, предложенному в [2], построим семейство операторов $R_{\alpha}=S_{\alpha} A^{-1}$.

Теорема 1. Операторы $R_{\alpha}$ являются интегральными операторами с ядрами $R_{\alpha}(x, t)$, имеющими вид

$$
R_{\alpha}(x, t)= \begin{cases}(\alpha \Gamma(1-\beta))^{-1} R_{\alpha 2}(x, t), & x \in[0,1 / 2], \\ (\alpha \Gamma(1-\beta))^{-1} R_{\alpha 1}(x, t), & x \in[1 / 2,1]\end{cases}
$$




$$
\begin{aligned}
& R_{\alpha 1}(x, t)= \begin{cases}(x-t)^{-\beta}-(x-\alpha-t)^{-\beta}, & 0 \leq t<x-\alpha, \\
(x-t)^{-\beta}, & x-\alpha \leq t<x, \\
0, & x \leq t \leq 1,\end{cases} \\
& R_{\alpha 2}(x, t)= \begin{cases}(x+\alpha-t)^{-\beta}-(x-t)^{-\beta}, & 0 \leq t<x, \\
(x+\alpha-t)^{-\beta}, & x \leq t<x+\alpha, \\
0, & x+\alpha \leq t \leq 1 .\end{cases}
\end{aligned}
$$

Доказательство основано на том, что в данном случае вид оператора ${ }^{-1}$ известен:

$$
A^{-1} f=\frac{d}{d x} \int_{0}^{x} \frac{(x-t)^{-\beta}}{\Gamma(1-\beta)} f(t) d t .
$$

Тогда из (2) и (6) следуют (3)-(5).

Теорема 2. Операторы $R_{\alpha_{j}}, j=1,2$, при $0<\beta<1 / 2$ являются линейными, ограниченныьми при каждом значении $\alpha$ операторами, действующими из пространства $L_{2}[0,1]$ в $C[1 / 2,1]$ при $j=1$ и в $C[0,1 / 2]$ при $j=2$. При этом справедлива двусторонняя оценка:

$$
C_{\beta} \alpha^{-\frac{2 \beta+1}{2}} \leq\left\|R_{\alpha}\right\|_{L_{2} \rightarrow L_{\infty}} \leq \sqrt{2} C_{\beta} \alpha^{-\frac{2 \beta+1}{2}},
$$

где $C_{\beta}=(\Gamma(1-\beta))^{-1}(1-2 \beta)^{-1 / 2},\|\cdot\|_{L_{\infty}}=\max \left\{\|\cdot\|_{C[0,1 / 2]},\|\cdot\|_{C[1 / 2,1]}\right\}$.

Доказательство. Из неравенства Буняковского следует, что операторы $R_{\alpha j}, j=1,2$, определены на всем пространстве $L_{2}[0,1]$ и ограничены при каждом фиксированном $\alpha$ и $0<\beta<1 / 2$. Действительно, например для $j=1$ и $t \in[x-\alpha, x)$ имеем:

$$
\left|\int_{x-\alpha}^{x}(x-t)^{-\beta} f(t) d t\right| \leq\left(\int_{x-\alpha}^{x}(x-t)^{-2 \beta} d t\right)^{1 / 2}\|f\|_{L_{2}}=(1-2 \beta)^{-1 / 2} \alpha^{1-2 \beta}\|f\|_{L_{2}} .
$$

Аналогичные оценки получаются для других интервалов изменения $t$ и также для $j=2$.

При этом значения интегральных операторов с ядрами $R_{\alpha j}(x, t)$, определенными в (4), (5), являются непрерывными функциями на соответствующих половинах отрезка $[0,1]$. Это следует из непрерывности функции $\int_{0}^{x}(x-t)^{-\beta} f(t) d t$, которая устанавливается при выводе формулы (6).

Далее, очевидно, что

$$
\left\|R_{\alpha}\right\|_{L_{2} \rightarrow L_{\infty}}=\max \left\{\left\|R_{\alpha 1}\right\|_{L_{2}[0,1] \rightarrow C[1 / 2,1]},\left\|R_{\alpha 2}\right\|_{L_{2}[0,1] \rightarrow C[0,1 / 2]}\right\} .
$$

При этом

$$
\begin{aligned}
& \left\|R_{\alpha 1}\right\|_{L_{2}[0,1] \rightarrow C 1 / 2,1}=[\alpha \Gamma(1-\beta)]^{-1} \max _{1 / 2 \leq x \leq 1}\left(\int_{0}^{1} R_{\alpha 1}^{2}(x, t) d t\right)^{1 / 2}, \\
& \left\|R_{\alpha 2}\right\|_{L_{2}[0,1] \rightarrow C 0,1 / 2}=[\alpha \Gamma(1-\beta)]^{-1} \max _{0 \leq x \leq 1 / 2}\left(\int_{0}^{1} R_{\alpha 2}^{2}(x, t) d t\right)^{1 / 2} .
\end{aligned}
$$

Рассмотрим сначала операторы $R_{\alpha 1}$. Подставив (4) в (9) и сделав замену переменных $x-t=\tau$, придем к выражению

$$
\int_{0}^{1} R_{\alpha 1}^{2}(x, t) d t=\int_{\alpha}^{x}\left[\tau^{-\beta}-(\tau-\alpha)^{-\beta}\right]^{2} d \tau+\int_{0}^{\alpha} \tau^{-2 \beta} d \tau
$$

Отсюда имеем:

$$
\int_{0}^{1} R_{\alpha 1}^{2}(x, t) d t \geq(1-2 \beta)^{-1} \alpha^{1-2 \beta}
$$


Далее, поскольку $\tau^{-\beta} \leq(\tau-\alpha)^{-\beta}$, то $\left[\tau^{-\beta}-(\tau-\alpha)^{-\beta}\right]^{2} \leq(\tau-\alpha)^{-2 \beta}-\tau^{-2 \beta}$, а отсюда следует:

$$
\int_{\alpha}^{x}\left[\tau^{-\beta}-(\tau-\alpha)^{-\beta}\right]^{2} d \tau \leq(1-2 \beta)^{-1}\left[(x-\alpha)^{1-2 \beta}-x^{1-2 \beta}+\alpha^{1-2 \beta}\right] .
$$

Поскольку $(x-\alpha)^{1-2 \beta} \leq x^{1-2 \beta}$, то в итоге приходим к оценке

$$
\int_{0}^{1} R_{\alpha 1}^{2}(x, t) d t \leq 2(1-2 \beta)^{-1} \alpha^{1-2 \beta} .
$$

Аналогично подставив (5) в (10) и сделав замену: $x+\alpha-t=\tau$, придем к оценкам (11), (12) для $\int_{0}^{1} R_{\alpha 2}^{2}(x, t) d t$.

Наконец, из (8)-(12) следует (7).

Следствие 1. Операторы $R_{\alpha}$ являются регуляризующими [3, с. 44] для уравнения (1).

Доказательство. Поскольку $R_{\alpha} A \equiv S_{\alpha}$, то требуемая для регуляризирующих операторов сходимость $\left\|R_{\alpha} A u-u\right\|_{L_{\infty}} \rightarrow 0$, где $u(x)-$ любая непрерывная функция, заданная на отрезке $[0,1]$, выполняется. Остальные требования к таким операторам устанавливаются в теореме 2 .

Рассмотрим величину

$$
\Delta\left(\delta, R_{\alpha}, u\right)=\sup \left\{\left\|R_{\alpha} f_{\delta}-u\right\|_{L_{\infty}}:\left\|f_{\delta}-f\right\|_{L_{2}} \leq \delta\right\} .
$$

Следствие 2. Для сходимости $\Delta\left(\delta, R_{\alpha}, u\right) \rightarrow 0$ при $\alpha \rightarrow 0, \delta \rightarrow 0$ необходимо и достаточно выполнения согласования $\alpha=\alpha(\delta)$ такого, ито $\alpha(\delta) \rightarrow 0$ и $\delta(\alpha(\delta))^{-\frac{2 \beta+1}{2}} \rightarrow 0$ при $\delta \rightarrow 0$.

Доказательство аналогично доказательству теоремы 1 из [4] с привлечением оценки (7).

2. Найдем конкретное согласование $\alpha=\alpha(\delta)$, обеспечивающее неулучшаемую по порядку оценку погрешности приближенных решений уравнения (1) в случае, когда $u(x) \in \operatorname{Lip}_{M} 1$.

Рассмотрим величину

$$
\Delta\left(\delta, R_{\alpha}, \operatorname{Lip}_{M} 1\right)=\sup \left\{\left\|R_{\alpha} f_{\delta}-u\right\|_{L_{\infty}}: u \in \operatorname{Lip}_{M} 1,\left\|f_{\delta}-f\right\|_{L_{2}} \leq 1\right\} .
$$

Теорема 3. Справедлива неулучшаемая по порядку $\delta$ оценка:

$$
\frac{1}{2} C_{1}(\beta) \delta^{\frac{2}{3+2 \beta}} \leq \Delta\left(\delta, R_{\alpha(\delta)}, \text { Lip }_{M} 1\right) \leq C_{2}(\beta) \delta^{\frac{2}{3+2 \beta}},
$$

где

$$
\begin{gathered}
\alpha(\delta)=D(\beta) \delta^{\frac{2}{3+2 \beta}}, \\
D(\beta)=\left(2^{1 / 2} M^{-1} C_{\beta}(2 \beta+1)\right)^{\frac{2}{3+2 \beta}}, \quad C_{1}(\beta)=\frac{M}{2} D(\beta)+C_{\beta}(D(\beta))^{-\frac{2 \beta+1}{2}},
\end{gathered}
$$

$C_{2}(\beta)$ отличается от $C_{1}(\beta)$ множителем 2 во втором слагаемом.

Доказательство. Пользуемся методом, приведенным в [5], и известной из теории некорректно поставленных задач оценкой:

$$
\frac{1}{2} \varphi(\alpha, \delta) \leq \Delta\left(\delta, R_{\alpha}, \operatorname{Lip}_{M} 1\right) \leq \varphi(\alpha, \delta),
$$

где

$$
\begin{gathered}
\varphi(\alpha, \delta)=\Delta_{1}\left(R_{\alpha} A, \operatorname{Lip}_{M} 1\right)+\delta\left\|R_{\alpha}\right\|_{L_{2} \rightarrow L_{\infty}}, \\
\Delta_{1}\left(R_{\alpha} A, \operatorname{Lip}_{M} 1\right)=\sup \left\{\left\|R_{\alpha} A u-u\right\|_{L_{\infty}}: u \in \operatorname{Lip}_{M} 1\right\} .
\end{gathered}
$$

Очевидно, что

$$
\Delta_{1}\left(R_{\alpha} A, \operatorname{Lip}_{M} 1\right) \equiv \Delta_{1}\left(S_{\alpha}, \operatorname{Lip}_{M} 1\right) .
$$


Далее, из условия Липшица следует оценка

$$
\Delta_{1}\left(S_{\alpha}, \operatorname{Lip}_{M} 1\right) \leq M \frac{\alpha}{2},
$$

которая достигается на функции $f_{0}(x)=M x$. Отсюда получаем равенство:

$$
\Delta_{1}\left(R_{\alpha} A, \operatorname{Lip}_{M} 1\right)=M \frac{\alpha}{2} .
$$

Из равенств (16), (17) и двусторонней оценки (7) получаем оценку:

$$
\Phi_{1}(\alpha, \delta) \leq \varphi(\alpha, \delta) \leq \Phi_{2}(\alpha, \delta),
$$

где $\Phi_{1}(\alpha, \delta)=M \frac{\alpha}{2}+C_{\beta} \alpha^{-\frac{2 \beta+1}{2}}$, а $\Phi_{2}(\alpha, \delta)$ отличается от $\Phi_{1}(\alpha, \delta)$ множителем $\sqrt{2}$ во втором слагаемом.

В соответствии с [5] найдем $\alpha=\alpha(\delta)$ из условия $\Phi_{2}(\alpha, \delta) \rightarrow \underset{\alpha}{\inf }$ и придем к формуле (14). Подставляя (14) в оценку (15), получаем оценку (13).

Сравнение регуляризации, проведенной здесь, с регуляризацией уравнения Абеля из [6] показывает, что в данном случае и семейство регуляризирующих операторов, и доказательства соответствующих теорем являются более простыми.

Работа выполнена при финансовой поддержке РФФИ (проект 13-01-00238).

\section{Библиограсрический список}

1. Хромов А. П., Хромова Г. В. Об одной модификации оператора Стеклова // Современные проблемы теории функций и их приложения : тез. докл. 15-й Сарат. зимн. школы. Саратов : Изд-во Сарат. ун-та, 2010. С. 181. 2. Хромова Г. В. Об одном способе построения методов регуляризации уравнений первого рода // Журн. вычисл. матем. и матем. физ. 2000. Т. 40, № 7. С. $997-$ 1002 .

3. Иванов В. К., Васин В. В., Танана В. П. Теория линейных некорректных задач и ее приложения. $M$. : Наука, 1978. 206 с.
4. Иванов В. К. Об интегральных уравнениях Фредгольма первого рода // Дифференц. уравнения. 1967. T. III, № 3. C. 410-421.

5. Хромова Г. В. Об оценках погрешности приближенных решений уравнений первого рода // Докл. АН. 2001. Т. 378, № 5. С. 605-609.

6. Хромова Г. В. О приближенных решениях уравнения Абеля // Вестн. Моск. ун-та. Сер. 15. 2001. № 3. C. $5-9$.

\section{Regularization of Abel Equation with the Use of Discontinuous Steklov Operator}

\section{G. V. Khromova}

Saratov State University, 83, Astrakhanskaya str., Saratov, 410012, Russia, KhromovAP@info.sgu.ru

For getting uniform approximations of the exact solution of Abel equation with an approximate right-hand part a simply constructed family of integral operators is suggested.

Key words: Abel equation, Steklov operator, uniform approximations, closed interval.

This work was supported by the Russian Foundation for Basic Research (project no. 13-01-00238).

\section{References}

1. Khromov A. P., Khromova G. V. Ob odnoi modifikatsii operatora Steklova [One modification of the Steklov operator]. Sovremennye problemy teorii funktsii $i$ ikh prilozheniia: Tez. dokl. 15-i Sarat. zimn. shkoly [Modern problems of function theory and their applications: abstracts of the 15-th Saratov winter school], Saratov, Saratov Univ. Press, 2010, pp. 181 (in Russian).

2. Khromova G. V. On a technique for constructing regularization methods for equations of the first kind.
Comput. Math. Math. Phys., 2000, vol. 40, no. 7, pp. 955-960.

3. Ivanov V. K., Vasin V. V., Tanana V. P. Teoriya lineinykh nekorrektnykh zadach $i$ ee prilozheniya [Theory of linear ill-posed problems and its applications]. Moscow, Nauka, 1978, 206 p.(in Russian).

4. Ivanov V. K. Ob integral'nykh uravneniiakh Fredgol'ma I roda [Fredholm integral equation of the first kind]. Differents. uravneniia [Differ. Equations], 1967, vol. III, no. 3, pp. 410-421 (in Russian). 
6. Khromova G. V. On the approximate solutions of the Abel's equation. Vestnik Moskovskogo universiteta.

Ser. 15, 2001, no. 4, pp. 5-9 (in Russian).
. Khromova G. V. Error estimates of approximate vol. 63 , no. $3,390-394$

\section{УДК 517.51}

\section{О ПРИБЛИЖЕНИИ И ВОССТАНОВЛЕНИИ НЕПРЕРЫВНЫХ ФУНКЦИЙ С КРАЕВЫМИ УСЛОВИЯМИ}

\section{О. И. Шаталина}

Специалист отдела расчетных операций, Локо-Банк, Саратов, OShatalina@ srt.lockobank.ru

В работе приведено семейство интегральных операторов, с помощью которых получаются равномерные приближения к непрерывной фрункции, удовлетворяющей краевым условиям (при этом указанные приближения удовлетворяют тем же условиям), и решена задача типа Колмогорова-Никольского на некотором компактном классе. Кроме того, с помощью полученного семейства интегральных операторов решается известная задача из теории некорректно поставленных задач, так называемая задача восстановления непрерывной срункци по ее среднеквадратичному приближению.

Ключевые слова: фрункционал Тихонова, семейство интегральных операторов, некорректно поставленная задача, задача типа Колмогорова - Никольского, равномерные приближения.

1. Пусть непрерывная функция $\bar{u}(x)$ удовлетворяет краевому условию:

$$
\cup(\bar{u}) \equiv \beta_{1} \bar{u}(0)+\beta_{2} \bar{u}(1)=0, \quad \beta_{1}^{2}+\beta_{2}^{2}>0 .
$$

Получим равномерные приближения к $\bar{u}(x)$, используя модификацию функционала Тихонова, известного в теории некорректно поставленных задач [1], а именно рассмотрим функционал

$$
M^{\alpha}[u, \bar{u}]=\|u-\bar{u}\|_{L_{2}}^{2}+\alpha\|u\|_{W_{2}^{1}}^{2},
$$

где $\alpha>0$ - параметр, $\|u\|_{W_{2}^{1}}=\left(\int_{0}^{1}\left(p u^{2}+\left(q u^{\prime}\right)^{2}\right) d x\right)^{1 / 2}, p, q-$ положительные константы. Это так называемый тихоновский функционал, но в данном случае он связывается не с интегральными уравнениями 1-го рода, как у А. Н. Тихонова, а с простейшим уравнением 1-го рода - уравнением с оператором вложения из пространства $C[0,1]$ в пространство $L_{2}[0,1]$. При этом будем считать допустимыми функциями функции, удовлетворяющие условию (1).

Обозначим через $u^{\alpha}(x)-$ функции, минимизирующие функционал (2) при каждом фиксированном значении $\alpha$. Существование этих функций при каждом фиксированном $\alpha$ доказывается точно так же, как в классической постановке А. Н. Тихонова [2].

Лемма 1. Минимизирующие функции и ${ }^{\alpha}(x)$ при каждом фиксированном а являются решением краевой задачи:

$$
\left\{\begin{array}{l}
-q y^{\prime \prime}+\left(p+\frac{1}{\alpha}\right) y=\frac{1}{\alpha} \bar{u} \\
\beta_{1} y(0)+\beta_{2} y(1)=0, \\
\beta_{2} y^{\prime}(0)+\beta_{1} y^{\prime}(1)=0 .
\end{array}\right.
$$

Доказательство. Рассмотрим функционал (2) и его приращение

$$
\triangle M^{\alpha}[u, \bar{u}]=M^{\alpha}\left[u^{\alpha}+\beta \eta, \bar{u}\right]-M^{\alpha}\left[u^{\alpha}, \bar{u}\right]
$$

где $\eta(x) \in W_{2}^{1}[0,1]$ и удовлетворяет условию $(1), \beta>0-$ произвольное вещественное число.

Сделав необходимые преобразования, учитывая свойства скалярного произведения, получаем:

$$
\triangle M^{\alpha}[u, \bar{u}]=2 \beta\left\{\left(u^{\alpha}-\bar{u}, \eta\right)_{L_{2}}+\alpha\left(u^{\alpha}, \eta\right)_{W_{2}^{1}}\right\}+\beta^{2}\left\{\|\eta\|_{L_{2}}^{2}+\alpha\|\eta\|_{W_{2}^{1}}^{2}\right\}
$$

Приравнивая линейную часть приращения функционала к нулю, получаем:

$$
\left(u^{\alpha}-\bar{u}, \eta\right)_{L_{2}}+\alpha\left(u^{\alpha}, \eta\right)_{W_{2}^{1}}=0 .
$$

